\title{
MORE PROPERTIES OF ALMOST COHEN-MACAULAY RINGS
}

\author{
CRISTODOR IONESCU
}

\begin{abstract}
Some interesting properties of almost CohenMacaulay rings are investigated, and a Serre type property connected with this class of rings is studied.
\end{abstract}

1. Introduction. A flaw in the first edition of [5] in the chapter dedicated to Cohen-Macaulay rings was corrected in the second edition. This led to the study of the so-called almost Cohen Macaulay rings, first by Han [1] and later by Kang [2, 3]. Since the first of these papers is written in Chinese, the others two are the main references for the subject.

Remark 1.1. Let $A$ be a commutative Noetherian ring, $P \in \operatorname{Spec}(A)$ and $M \neq 0$ a finitely generated $A$-module. Then $\operatorname{depth}_{P}(M) \leq$ $\operatorname{depth}_{P A_{P}} M_{P}$.

Definition 1.2. (cf. $[\mathbf{1}, \mathbf{2}]$ ). Let $A$ be a commutative Noetherian ring. A finitely generated $A$-module $M \neq 0$ is called almost Cohen-Macaulay if $\operatorname{depth}_{P} M=\operatorname{depth}_{P A_{P}} M_{P}$, for any $P \in \operatorname{Supp}(M) . A$ is called an almost Cohen-Macaulay ring if it is an almost Cohen-Macaulay $A$ module, that is, if for any $P \in \operatorname{Spec}(A), \operatorname{depth}_{P} A=\operatorname{depth}_{P A_{P}} A_{P}$.

Several properties of almost Cohen-Macaulay rings are proved in [2], and several interesting examples are given in [3]. In the following, we are trying to complete the results in [2] and to introduce a Serre-type condition that we call $\left(C_{k}\right)$, for any $k \in \mathbb{N}$ condition that is to be to almost Cohen-Macaulay rings what the classical Serre condition $\left(S_{k}\right)$ is to Cohen-Macaulay rings.

2010 AMS Mathematics subject classification. Primary 13C14, 13H10.

Keywords and phrases. almost Cohen-Macaulay modules, Serre property.

Received by the editors on March 3, 2014, and in revised form on May 16, 2014. DOI:10.1216/JCA-2015-7-3-363 Copyright (C)2015 Rocky Mountain Mathematics Consortium 
2. Properties of almost Cohen-Macaulay rings. All rings considered will be commutative and with unit. We start by reminding the reader about some basic properties of almost Cohen-Macaulay rings.

Remark 2.1. Let $A$ be a Noetherian ring. Then:

(a) $A$ is almost Cohen-Macaulay if and only if ht $(P) \leq 1+\operatorname{depth}_{P} A$, for all $P \in \operatorname{Spec}(A)([2,1.5])$;

(b) $A$ is almost Cohen-Macaulay if and only if $A_{P}$ is almost CohenMacaulay for any $P \in \operatorname{Spec}(A)$ if and only if $A_{Q}$ is almost CohenMacaulay for any $Q \in \operatorname{Max}(A)$ if and only if ht $(Q) \leq 1+\operatorname{depth} A_{Q}$ for any $Q \in \operatorname{Max}(A)([2,2.6])$;

(c) If $A$ is local, it follows from b) that $A$ is almost Cohen-Macaulay if and only if $\operatorname{dim}(A) \leq 1+\operatorname{depth}(A)$.

Our first result is a stronger formulation of $[\mathbf{2}, 2.10]$ and deals with the behavior of almost Cohen-Macaulay rings with respect to flat morphisms.

Proposition 2.2. Let $u:(A, m) \rightarrow(B, n)$ be a local flat morphism of Noetherian local rings.

(a) If $B$ is almost Cohen-Macaulay, then $A$ and $B / m B$ are almost Cohen-Macaulay.

(b) If $A$ and $B / m B$ are almost Cohen-Macaulay and one of them is Cohen-Macaulay, then $B$ is almost Cohen-Macaulay.

Proof.

(a) We have

$$
\begin{aligned}
\operatorname{dim}(A) & =\operatorname{dim}(B)-\operatorname{dim}(B / m B) \leq 1+\operatorname{depth} B-\operatorname{dim}(B / m B) \\
& \leq 1+\operatorname{depth} B-\operatorname{depth}(B / m B)=1+\operatorname{depth} A .
\end{aligned}
$$

We also have

$$
\begin{aligned}
\operatorname{dim}(B / m B)-\operatorname{depth}(B / m B)= & (\operatorname{dim}(B)-\operatorname{depth} B) \\
& -(\operatorname{dim}(A)-\operatorname{depth} A) \\
\leq & 1-(\operatorname{dim}(A)-\operatorname{depth} A) \leq 1 .
\end{aligned}
$$


(b) Since $u$ is flat, we have

$$
\begin{aligned}
\operatorname{dim}(B) & =\operatorname{dim}(A)+\operatorname{dim}(B / m B) \leq 1+\operatorname{depth}(A)+\operatorname{depth}(B / m B) \\
& =1+\operatorname{depth}(B) .
\end{aligned}
$$

Question 2.3. We do not know of any example of a local flat morphism of Noetherian local rings $u:(A, m) \rightarrow(B, n)$ such that $A$ and $B / m B$ are almost Cohen-Macaulay and $B$ is not almost Cohen-Macaulay. ${ }^{1}$

Corollary 2.4. Let $A$ be a Noetherian local ring, $I \neq A$ an ideal contained in the Jacobson radical of $A$ and $\widehat{A}$ the completion of $A$ in the I-adic topology. Then $A$ is almost Cohen-Macaulay if and only if $\widehat{A}$ is almost Cohen-Macaulay.

Proof. Since $I$ is contained in the Jacobson radical of $A$, the canonical morphism $A \rightarrow \widehat{A}$ is faithfully flat and $\operatorname{Max}(A) \cong \operatorname{Max}(\widehat{A})$. Moreover, if $m \in \operatorname{Max}(A)$ and $\widehat{m}$ is the corresponding maximal ideal of $\widehat{A}$, the closed fiber of the morphism $A_{m} \rightarrow \widehat{A}_{\hat{m}}$ is a field. Now apply Proposition 2.2.

Corollary 2.5. (see $[\mathbf{2}, 1.6]$ ). Let $A$ be a Noetherian ring and $n \in \mathbb{N}$. Then $A$ is almost Cohen-Macaulay if and only if $A\left[\left[X_{1}, \ldots, X_{n}\right]\right]$ is almost Cohen-Macaulay.

Proof. Suppose that $A$ is almost Cohen-Macaulay. We may clearly assume that $A$ is local and $n=1$. By $[2,1.3]$, we get that $A[X]_{(X)}$ is almost Cohen-Macaulay. Now apply Corollary 2.4. The converse is clear.

For the next corollary, we need some notation.

Notation 2.6. If $\mathbf{P}$ is a property of Noetherian local rings, we denote by $\mathbf{P}(A):=\left\{Q \in \operatorname{Spec}(A) \mid A_{Q}\right.$ has the property $\left.\mathbf{P}\right\}$ and by $\mathbf{N P}(A):=\left\{Q \in \operatorname{Spec}(A) \mid A_{Q}\right.$ does not have the property $\left.\mathbf{P}\right\}=$ $\operatorname{Spec}(A) \backslash \mathbf{P}(A)$. 
Definition 2.7. Let $A$ be a Noetherian ring. According to Notation 2.6, the set

$\operatorname{aCM}(A):=\left\{P \in \operatorname{Spec}(A) \mid A_{P}\right.$ is almost Cohen-Macaulay $\}$

is called the almost Cohen-Macaulay locus of $A$.

Corollary 2.8. Let $u: A \rightarrow B$ be a morphism of Noetherian local rings and $\varphi: \operatorname{Spec}(B) \rightarrow \operatorname{Spec}(A)$ the induced morphism on the spectra. If the fibers of $u$ are Cohen-Macaulay, then $\varphi^{-1}(\mathbf{a C M}(A))=\mathbf{a C M}(B)$.

Proof. Obvious from Proposition 2.2.

In Cohen-Macaulay rings, chains of prime ideals behave very well, in the sense that Cohen-Macaulay rings are universally catenary (see [5]). This is no longer the case for almost Cohen-Macaulay rings.

Example 2.9. There exists a local almost Cohen-Macaulay ring which is not catenary.

Proof. Indeed, by [2, Example 2], any Noetherian normal integral domain of dimension 3 is almost Cohen-Macaulay. In [6], such a ring which is not catenary is constructed.

The next result shows that some of the formal fibers of almost CohenMacaulay rings are almost Cohen-Macaulay. A stronger fact will be proved in Proposition 2.13.

Proposition 2.10. Let $A$ be a Noetherian local almost Cohen-Macaulay ring, $P \in \operatorname{Spec}(A), Q \in \operatorname{Ass}(\widehat{A} / P \widehat{A})$. Then $\widehat{A}_{Q} / P \widehat{A}_{Q}$ is almost CohenMacaulay.

Proof. We have

$$
\begin{aligned}
\operatorname{dim}\left(\widehat{A}_{Q} / P \widehat{A}_{Q}\right) & =\operatorname{dim} \widehat{A}_{Q}-\operatorname{dim} A_{P} \\
& \leq \operatorname{depth} \widehat{A}_{Q}+1-\operatorname{dim} A_{P} \\
& \leq \operatorname{depth} \widehat{A}_{P}+1-\operatorname{dim} A_{P} \\
& =\operatorname{depth}\left(\widehat{A}_{Q} / P \widehat{A}_{Q}\right)+1 .
\end{aligned}
$$


The following result shows that the almost Cohen-Macaulay property is preserved by tensor products and finite field extensions.

Proposition 2.11. Let $k$ be a field and $A$ and $B$ two $k$-algebras such that $A \otimes_{k} B$ is a Noetherian ring. If $A$ and $B$ are almost CohenMacaulay and one of them is Cohen-Macaulay, then $A \otimes_{k} B$ is almost Cohen-Macaulay.

Proof. Let $P \in \operatorname{Spec}(A)$. We have a flat morphism $B \rightarrow B \otimes_{k} k(P)$. Let $Q \in \operatorname{Spec}(B)$. Set $T:=A / P \otimes_{k} B / Q=A \otimes_{k} B /\left(P \otimes_{k} B+A \otimes_{k} Q\right)$. Then $k(P) \otimes_{k} k(Q)$ is a ring of fractions of $T$, hence Noetherian by assumption. By [7, Proposition 5], it follows that $k(P) \otimes_{k} k(Q)$ is locally a complete intersection. Now let $Q \in \operatorname{Spec}(B)$ and $P=Q \cap A$. By the above, the flat local morphism $A_{P} \rightarrow\left(B \otimes_{k} k(P)\right)_{Q}$ has a complete intersection closed fiber; hence, the ring $\left(B \otimes_{k} k(P)\right)_{Q}$ is almost Cohen-Macaulay by Proposition 2.2. Now consider the flat morphism $A \rightarrow A \otimes_{k} B$ and let $Q \in \operatorname{Spec}\left(A \otimes_{k} B\right)$ and $P=Q \cap A$. Then the flat local morphism $A_{P} \rightarrow\left(A \otimes_{k} B\right)_{Q}$ has a complete intersection closed fiber, whence $\left(A \otimes_{k} B\right)_{Q}$ is almost Cohen-Macaulay.

Corollary 2.12. Let $k$ be a field, $A$ a Noetherian $k$-algebra which is almost Cohen-Macaulay and $L$ a finite field extension of $k$. Then $A \otimes_{k} L$ is almost Cohen-Macaulay.

As for the Cohen-Macaulay property, the formal fibers of factorizations of almost Cohen-Macaulay rings are almost Cohen-Macaulay.

Proposition 2.13. Let $B$ be a local almost Cohen-Macaulay ring, $I$ an ideal of $B$ and $A=B / I$. Then the formal fibers of $A$ are almost Cohen-Macaulay.

Proof. We have $\widehat{A}=\widehat{B} \otimes_{B} A=\widehat{B} / I \widehat{B}$; hence, the formal fibers of $A$ are exactly the formal fibers of $B$ in the prime ideals of $B$ containing $I$. Let $P$ be such a prime ideal, let $S=B \backslash P$ and let $C:=S^{-1}(\widehat{B} / I \widehat{B})$. Also let $Q \in \operatorname{Spec}(C)$. There exists $Q^{\prime} \in \operatorname{Spec}(\widehat{B})$ such that $Q=Q^{\prime} C$ and $Q^{\prime} \cap B=P$. Thus, we have a local flat morphism $B_{Q} \rightarrow \widehat{B}_{Q^{\prime}}$. But $B$ is almost Cohen-Macaulay; hence, $\widehat{B}_{Q^{\prime}}$ 
and consequently $C_{Q} \cong \widehat{B}_{Q^{\prime}} / P \widehat{B}_{Q^{\prime}}$ are almost Cohen-Macaulay, by Proposition 2.2.

3. The property $\left(C_{n}\right)$. Recall that, given a natural number $n$, a Noetherian ring $A$ is said to have Serre property $\left(S_{n}\right)$ if $\operatorname{depth}\left(A_{P}\right) \geq$ $\min ($ ht $P, n)$ for any prime ideal $P \in \operatorname{Spec}(A)$. Moreover, $A$ is CohenMacaulay if and only if $A$ has the property $\left(S_{n}\right)$ for any $n \in \mathbb{N}$ (see [5, (17.I)]). We will try to characterize almost Cohen-Macaulay rings in a similar way.

Definition 3.1. Let $n \in \mathbb{N}$ be a natural number. We say that a Noetherian ring $A$ has the property $\left(C_{n}\right)$ if $\operatorname{depth}\left(A_{P}\right) \geq \min ($ ht $P, n)-$ 1 , for all $P \in \operatorname{Spec}(A)$.

Remark 3.2. (a) It is clear that $\left(C_{n}\right) \Rightarrow\left(C_{n-1}\right)$ and that $\left(S_{n}\right) \Rightarrow$ $\left(C_{n}\right)$, for all $n \in \mathbb{N}$.

(b) It is also clear that if $A$ has $\left(C_{n}\right)$, then $A_{P}$ has $\left(C_{n}\right)$, for all $P \in \operatorname{Spec}(A)$.

Theorem 3.3. A Noetherian ring $A$ is almost Cohen-Macaulay if and only if $A$ has the property $\left(C_{n}\right)$ for every $n \in \mathbb{N}$.

Proof. Assume that $A$ is almost Cohen-Macaulay, and let $P \in$ $\operatorname{Spec}(A)$. Then $A_{P}$ is almost Cohen-Macaulay; hence, $\operatorname{depth}\left(A_{P}\right) \geq$ ht $(P)-1$. If $n \geq$ ht $(P)$, then $\min ($ ht $(P), n)=$ ht $(P)$. Hence, $\operatorname{depth}\left(A_{P}\right) \geq \min (n$, ht $(P))-1$. If $n<$ ht $(P)$, then $\min (n$, ht $(P))=n$, so that $\operatorname{depth}\left(A_{P}\right) \geq \operatorname{ht}(P)-1>n-1=\min ($ ht $(P), n)-1$.

For the converse, let $P \in \operatorname{Spec}(A)$, ht $(P)=l$. Then

$$
\operatorname{depth}\left(A_{P}\right) \geq \min (l, \text { ht }(P))-1=\operatorname{ht}(P)-1 .
$$

Proposition 3.4. Let $k \in \mathbb{N}$. A Noetherian ring $A$ has the property $\left(C_{k}\right)$ if and only if $A_{P}$ is almost Cohen-Macaulay for any $P \in \operatorname{Spec}(A)$ with $\operatorname{depth}\left(A_{P}\right) \leq k-2$.

Proof. Let $P \in \operatorname{Spec}(A)$ be such that $\min (k$, ht $(P))-1 \leq \operatorname{depth}\left(A_{P}\right) \leq$ $k-2$. If ht $(P) \leq k$, then $\operatorname{depth}\left(A_{P}\right) \geq$ ht $(P)-1$. And, if ht $(P)>k$, then it follows that $k-2>\operatorname{depth}\left(A_{P}\right) \geq k-1$. This is a contradiction. 
Conversely, let $P \in \operatorname{Spec}(A)$. If $\operatorname{depth}\left(A_{P}\right) \leq k-2$, then $A_{P}$ is almost Cohen-Macaulay, hence ht $(P)-1 \leq \operatorname{depth}\left(A_{P}\right) \leq k-2$. Thus, $\min ($ ht $(P), k)=$ ht $(P)$, whence $\operatorname{depth}\left(A_{P}\right) \geq \min (k$, ht $(P)$. If $k-2<\operatorname{depth}\left(A_{P}\right)$, then ht $(P)>k-2$. Hence, $\operatorname{depth}\left(A_{P}\right) \geq$ $\min (k$, ht $(P))-1$.

Proposition 3.5. Let $A$ be a Noetherian ring, $k \in \mathbb{N}$ and $x \in A$ a non zero divisor. If $A / x A$ has the property $\left(C_{k}\right)$, then $A$ has the property $\left(C_{k}\right)$.

Proof. Let $Q \in \operatorname{Spec}(A)$ be such that $\operatorname{depth}\left(A_{Q}\right)=n \leq k-2$. If $x \in$ $Q$, then $\operatorname{depth}(A / x A)_{Q}=n-1 \leq k-3$. Then ht $(Q / x A) \leq n-1+1=$ $n$; hence, ht $(Q) \leq n+1=\operatorname{depth} A_{Q}+1$. If $x \notin Q$, let $P \in \operatorname{Min}(Q+x A)$. Then $(Q+x A) A_{P}$ is $P A_{P}$-primary and $\operatorname{depth}\left(A_{P}\right) \leq \operatorname{depth}\left(A_{Q}\right)+1=$ $n+1$. Then $\operatorname{depth}(A / x A)_{Q}=n-1$; hence, ht $(P / x A) \leq n$. It follows that ht $(P) \leq n+1=\operatorname{depth}\left(A_{P}\right)+1$.

Definition 3.6. We say that a property $\mathbf{P}$ of Noetherian local rings satisfies Nagata's criterion (NC) if the following holds: if $A$ is a Noetherian ring such that, for every $P \in \mathbf{P}(A)$, the set $\mathbf{P}(A / P)$ contains a non-empty open set of $\operatorname{Spec}(A / P)$, then $\mathbf{P}(A)$ is open in $\operatorname{Spec}(A)$.

An interesting study of the Nagata criterion is performed in [4].

Theorem 3.7. Let $k \in \mathbb{N}$. Property $\left(C_{k}\right)$ satisfies (NC).

Proof. Let $Q \in C_{k}(A)$. Then $\operatorname{depth}\left(A_{Q}\right) \geq \min (k$, ht $(Q))-1$.

Case a). ht $(Q) \leq k$. Then $\min (k$, ht $(Q))=$ ht $(Q)$; hence, $\operatorname{depth}\left(A_{Q}\right)+1 \geq \operatorname{ht}(Q)$ and $A_{Q}$ is almost Cohen-Macaulay. Let $f \in A \backslash Q$ be such that

$$
\operatorname{dim}\left(A_{P}\right)=\operatorname{dim}\left(A_{Q}\right)+\operatorname{dim}\left(A_{P} / Q A_{P}\right)
$$

and

$$
\operatorname{depth}\left(A_{P}\right)=\operatorname{depth}\left(A_{Q}\right)+\operatorname{depth}\left(A_{P} / Q A_{P}\right)
$$

for any $P \in D(f) \cap V(Q) \cap N T_{k}(A)$. Then

$$
\operatorname{depth}\left(A_{P}\right) \not \min (k, \operatorname{ht}(P))-1 .
$$


Case a1). ht $(P) \leq k$. Then $\min (k$, ht $(P))=$ ht $(P)$; hence, $\operatorname{depth}\left(A_{P}\right)+1<\operatorname{ht}(P)$. Then

$$
\begin{aligned}
\operatorname{depth}\left(A_{P} / Q A_{P}\right)+1 & =\operatorname{depth}\left(A_{P}\right)-\operatorname{depth}\left(A_{Q}\right)+1 \\
& <\operatorname{ht}(P)-\operatorname{depth}\left(A_{Q}\right) \leq \operatorname{ht}(P)-\operatorname{ht}(Q)+1 .
\end{aligned}
$$

Then $\operatorname{depth}\left(A_{P} / Q A_{P}\right)<\operatorname{dim}\left(A_{P} / Q A_{P}\right)=\operatorname{dim}\left(A_{P}\right)-\operatorname{dim}\left(A_{Q}\right)$, and it follows that $A_{P} / Q A_{P}$ is not $\left(C_{k}\right)$.

Case a2). ht $(P)>k$. Then $\min (k$, ht $(P))=k$; hence, $\operatorname{depth}\left(A_{P}\right)<$ $k-1$. It follows that

$$
\begin{aligned}
\operatorname{depth}\left(A_{P} / Q A_{P}\right) & =\operatorname{depth}\left(A_{P}\right)-\operatorname{depth}\left(A_{Q}\right) \\
& <k-1+1-\operatorname{ht}(Q)=k-\operatorname{ht}(Q) .
\end{aligned}
$$

This implies that $A_{P} / Q A_{P}$ is not $\left(C_{k}\right)$.

Case b). ht $(Q)>k$. Then $\min (k$, ht $(Q))=k$ and $\operatorname{depth}\left(A_{Q}\right)+$ $1 \geq k$. Since ht $(P)>k$, it follows that $\min (k$, ht $(P))=k$ and $\operatorname{depth}\left(A_{P}\right)+1<k$. Let $x_{1}, \ldots, x_{r}$ be an $A_{Q}$-regular sequence. Then there exists $f \in A \backslash Q$ such that $x_{1}, \ldots, x_{r}$ is $A_{f}$-regular. If $P \in D(f) \cap V(Q)$, it follows that $A_{P}$ is $\left(C_{k}\right)$.

Corollary 3.8. The property almost Cohen-Macaulay satisfies (NC).

Theorem 3.9. Let $A$ be a quasi-excellent ring and $k \in \mathbb{N}$. Then $C_{k}(A)$ and $\mathbf{a C M}(A)$ are open in the Zariski topology of $\operatorname{Spec}(A)$.

Proof. Let $P \in \operatorname{Spec}(A)$. Then $\operatorname{aCM}(A / P)$ and $C_{k}(A / P)$ contain the non-empty open set $\boldsymbol{R e g}(A / P)=\left\{P \in \operatorname{Spec}(A) \mid A_{P}\right.$ is regular $\}$. Now apply Theorems 3.7 and 3.8 .

Corollary 3.10. Let $A$ be a complete semilocal ring and $k \in \mathbb{N}$. Then $C_{k}(A)$ and $a C M(A)$ are open in the Zariski topology of $\operatorname{Spec}(A)$.

Corollary 3.11. Let $A$ be a Noetherian local ring with CohenMacaulay formal fibers. Then aCM $(A)$ is open.

Proof. Follows from Corollaries 2.8 and 3.10. 
Proposition 3.12. Let $u: A \rightarrow B$ be a flat morphism of Noetherian rings and $k \in \mathbb{N}$. If $B$ has $\left(C_{k}\right)$, then $A$ has $\left(C_{k}\right)$.

Proof. We may assume that $A$ and $B$ are local rings and that $u$ is local. Let $P \in \operatorname{Spec}(A)$ and $Q \in \operatorname{Min}(P B)$. Then $\operatorname{dim}\left(B_{Q} / P B_{Q}\right)=0$; hence,

$$
\begin{aligned}
\operatorname{depth}\left(A_{P}\right) & =\operatorname{depth}\left(B_{Q}\right) \geq \min \left(k, \operatorname{dim}\left(B_{Q}\right)\right)-1 \\
& =\min \left(k, \operatorname{dim}\left(A_{P}\right)\right)-1
\end{aligned}
$$

Proposition 3.13. Let $u: A \rightarrow B$ be a flat morphism of Noetherian rings and $k \in \mathbb{N}$.

a) If $A$ has $\left(C_{k}\right)$ and all the fibers of $u$ have $\left(S_{k}\right)$, then $B$ has $\left(C_{k}\right)$.

b) If $A$ has $\left(S_{k}\right)$ and all the fibers of $u$ have $\left(C_{k}\right)$, then $B$ has $\left(C_{k}\right)$.

Proof. a) Let $Q \in \operatorname{Spec}(B), P=Q \cap A$. Then, by flatness, we have

$$
\begin{aligned}
\operatorname{dim}\left(B_{Q}\right) & =\operatorname{dim}\left(A_{P}\right)+\operatorname{dim}\left(B_{Q} / P B_{Q}\right), \\
\operatorname{depth}\left(B_{Q}\right) & =\operatorname{depth}\left(A_{P}\right)+\operatorname{depth}\left(B_{Q} / P B_{Q}\right) .
\end{aligned}
$$

By assumption, we have

$$
\begin{aligned}
\operatorname{depth}\left(A_{P}\right) & \geq \min (k, \operatorname{ht}(P))-1, \\
\operatorname{depth}\left(B_{Q} / P B_{Q}\right) & \geq \min \left(k, \operatorname{dim}\left(B_{Q} / P B_{Q}\right) .\right.
\end{aligned}
$$

Hence, we have

$$
\begin{aligned}
\operatorname{depth}\left(B_{Q}\right)= & \operatorname{depth}\left(A_{P}\right)+\operatorname{depth}\left(B_{Q} / P B_{Q}\right) \\
\geq & \min (k, \operatorname{ht}(P))-1 \\
& +\min \left(k, \operatorname{dim}\left(B_{Q} / P B_{Q}\right)\right) \\
& =\min \left(k, \operatorname{ht}\left(B_{Q}\right)\right)-1
\end{aligned}
$$

b) The proof is the same.

As a corollary we get a new proof of a previous result.

Corollary 3.14. Let $u: A \rightarrow B$ be a flat morphism of Noetherian rings.

a) If $B$ is almost Cohen-Macaulay, then $A$ is almost Cohen-Macaulay. 
b) If $A$ is almost Cohen-Macaulay and the fibers of $u$ are CohenMacaulay, then $B$ is almost Cohen-Macaulay.

Example 3.15. Let $k$ be a field, and let $X_{0}, X_{1}, X_{2}, Y_{1}, Y_{2}$ be indeterminates. Set $B=k\left[\left[X_{0}, X_{1}, X_{2}\right]\right] /\left(X_{0}\right) \cap\left(X_{0}, X_{1}\right)^{2} \cap\left(X_{0}, X_{1}, X_{2}\right)^{3}$ and $A:=B\left[\left[Y_{1}, Y_{2}\right]\right]$. It is easy to see that $A$ is a Noetherian local ring with $\operatorname{dim}(A)=5, \operatorname{depth}(A)=2$. It is also not difficult to see that $A$ has property $\left(C_{3}\right)$ and not property $\left(C_{4}\right)$. Other similar examples can easily be constructed.

Example 3.16. Let $k$ be a field, $X$ and $Y$ indeterminates and consider the ring $A=k[[X, Y]] /\left(X^{2}, X Y\right)$. Then $A$ has $\left(C_{2}\right)$ and not $\left(S_{2}\right)$.

\section{ENDNOTES}

1. An example was given by M. Tabaâ, Sur le produit tensoriel d'algèbres, preprint, arXiv:1304.5395.

\section{REFERENCES}

1. Y. Han, D-rings, Acta Math. Sinica 4 (1998), 1047-1052.

2. M.C. Kang, Almost Cohen-Macaulay modules, Comm. Alg. 29 (2001), 781787 .

3. _ Addendum to "Almost Cohen-Macaulay modules," Comm. Alg. 30 (2002), 1049-1052.

4. C. Massaza, Su alcuni aspetti assiomatici del criterio di Nagata, Rend. Circ. Mat. Palermo 30 (1981), 365-377.

5. H. Matsumura, Commutative algebra, 2nd edition, Benjamin Cummings, 1980.

6. T. Ogoma, Non-catenary pseudo-geometric normal rings, Japan J. Math. 6 (1980), 147-163.

7. M. Tousi and S. Yassemi, Tensor products of some special rings, J. Algebra 268 (2003), 672-675.

Institute of Mathematics, Simion Stollow of the Romanian Academy, P.O. Box 1-764, RO 014700 Bucharest, ROMANIA

Email address: cristodor.ionescu@imar.ro 